\title{
Stereotactic Body Radiation Therapy in Hepatocellular Carcinoma
}

Hepatocellular carcinoma (HCC) is a primary liver cancer believed to originate from primary stem cells. ${ }^{[1]}$ This cancer is increasing in its incidence likely due to the increase in the incidence of chronic hepatitis. ${ }^{[2]}$ The treatment of choice is surgery and therefore every patient must undergo evaluation for surgery. However, most patients present in an inoperable stage ${ }^{[3]}$ and the intent of treatment becomes palliative, an important goal of therapy being preserving the liver function for as long as possible. To achieve this, many liver-directed therapies (LDTs) have come up that can stall tumor progression. These include transarterial chemoembolization (TACE), transarterial radioembolization, radiofrequency ablation (RFA), intra-arterial chemotherapy, targeted drugs (sorafenib and lenvatinib), and IO drugs (nivolumab and pembrolizumab). In this therapeutic armamentarium, stereotactic body radiation therapy (SBRT) is an important addition. There is no specific experimental data that compare and contrast the above-mentioned modalities, and therefore, the treatment offered at most institutions is largely empirical and dependent on expertise and preferences of treating team. In this paper, we attempt to delineate the merits and relative strengths of SBRT for HCC.

SBRT is defined as a method of external beam radiotherapy that accurately delivers a high radiation dose to a target in one or few treatment fractions. ${ }^{[4]}$ It is done by focusing high-energy radiation beams from multiple directions via specialized linear accelerators (Trubeam, Trilogy, Novalis, Edge, Synergy, Cyberknife, and others) that provide precise delivery of dose to prespecified target by shaping of beam perfectly on to the target and by checking for even minor errors in the position of target at the time of radiation delivery by image guidance technology. Typically, SBRT treatment sessions are 3-10 in number, and the dose delivered in each fraction is 5 Gy or more depending on the best compromise reached between radiation dose to the target and normal tissues on the computerized planning system. Smaller the tumor, larger the dose that can be delivered into it without compromising liver function. The aim is to ablate the tumor without damage to the functioning liver tissue.

Let us examine the role of SBRT in various clinical situations with HCC.

\section{For Small Lesions}

Small lesions are typically those that are $<3 \mathrm{~cm}$ in size. While RFA is a valuable modality in such cases, SBRT has been reported to provide equivalent results in some studies ${ }^{[5]}$ and is deemed inferior in others. ${ }^{[6]}$ In general, we can expect $90 \%-100 \%$ local control (LC), which is equivalent to RFA.
LC may be lesser for patients with worse baseline liver function, such as Child-Turcotte-Pugh (CP)-B patients. The same is likely related to the lower radiation dose delivered in order to decrease the risk of radiation-induced liver disease (RILD) in these patients. ${ }^{[7-10]}$ However, RFA studies report more long-term data which are scarce with regard to SBRT, being a relatively newer modality. Wahl et al. published a retrospective study and reported a comparison between SBRT and RFA. They reported that the results tilt in favor of SBRT once the size increases above $2 \mathrm{~cm} .{ }^{[5]}$ SBRT seems to be an equivalent modality to RFA at sizes below that and will be preferable in lesions close to blood vessels, subcapsular location, the nonspherical shape of borderline size, or diffuse lesions with no clear margins, all of which are tumor-related factors that predict higher rates of failure with RFA. ${ }^{[1]}$

\section{Stereotactic Body Radiation Therapy in Locally Advanced Hepatocellular Carcinoma}

In 2006, Méndez Romero et al. reported the first prospective data on liver SBRT for HCC demonstrating LC and safety in 11 lesions. LC rates at 1 and 2 years for the whole group (which included liver metastases) were 94\% and $82 \%, 1$ CP-B patient experienced RILD-related death. ${ }^{[12]}$ Kang et al. reported a prospective phase II trial in 56 HCC lesions, showing 2-year LC of $95 \%$ after SBRT. Grade $\geq 3$ toxicity was reported at $4.3 \% \cdot{ }^{[13]}$ Bujold et al. ${ }^{[14]}$ reported an analysis of 102 patients with HCC (CP-A 100\%) and reported a 1 -year LC of $87 \%$. The lesions treated were a median diameter of $7.2 \mathrm{~cm}$ (range: $1.4-23.1 \mathrm{~cm}$ ). Hepatotoxicity was seen in $30 \%$ of patients. Seven percent of deaths were possibly related to RILD. Culleton et al. ${ }^{[15]}$ published a prospective study on 29 patients with $\mathrm{HCC}$ with CP-B7 and above (69\% B7). Portal venous tumor thrombus (PVTT) was present in $76 \%$. They reported LC of $65 \%$ at 1 year, which is lower than previous studies and a median OS of 8 months. Ten patients had locally advanced HCC that was in primarily life-threatening stage, e.g., PVTT. They reported that patients with CP-B7 have a significantly higher median OS of 9.9 months versus 2.8 months in CP-B8 + patients $(P=0.011)$. They also showed that $\mathrm{CP} \geq \mathrm{B} 8$ and alpha fetoprotein $>4491 \mathrm{ng} / \mathrm{mL}$ were poor prognostic factors. All these suggest that patients with CP-B8 + should not be treated with SBRT as it may not improve outcomes. They reported a higher rate of hepatotoxicity with a CP-score increase of 2 or greater $(\mathrm{CP}+2)$ of $63 \%$ at 3 months. These studies show that patients often referred for SBRT are large, pretreated with other LDTs, often having PVTT and therefore patients need to be chosen wisely based on size and $\mathrm{CP}$ score balanced with the expected goal of treatment 
(for example, in some very large-sized cases, treating only PVTT can yield good outcomes with a possibly lower risk of hepatotoxicity). Furthermore, the presence of so many adverse factors compounded with pretreatment with other LDTs may confound LC, intrahepatic control, and hepatotoxicity reporting. ${ }^{[13,14,16]}$

\section{Stereotactic Body Radiation Therapy Along with Transarterial Chemoembolization}

This deserves a special mention because the most common pretreatment for HCC patients referred for SBRT is TACE with $40 \%-100 \%$ patients having received the same. ${ }^{[13-16]}$ Huo and Eslick reported a meta-analysis of 17 trials with a total of 25 trials (11 RCTs) involving 2577 patients. TACE plus RT showed significantly better 1-year survival and complete response rate compared with TACE alone. The survival benefit progressively increased for 2-, 3-, 4-, and 5-year survival. The authors concluded that for an inoperable HCC patient, TACE plus RT was more therapeutically beneficial than TACE alone. ${ }^{[17]}$ Since there are no set guidelines, SBRT and TACE are either utilized together in a planned manner or only as salvage after TACE. Buckstein et al. reported a retrospective review of outcomes in 103 patients receiving SBRT within 2 years following TACE to a target lesion. Fifty-two patients had planned adjuvant SBRT after TACE, and the remainder had salvage SBRT with no significant differences between the characteristics of the two groups. Planned TACE + SBRT reported more $\mathrm{CR}$ rates than salvage SBRT (79.6\% vs. $43.5 \%, P=0.006)$. One-year survival for planned TACE SBRT was $70.8 \%$ versus $61.5 \%$ for salvage $(P=0.052)$. They concluded that TACE + SBRT might achieve superior outcomes than salvage in view of superior overall response and CR rates. ${ }^{[18]}$

SBRT for PVTT: Incidence of PVTT in cases of HCC can be as high as $40 \% .^{[19]}$ The presence of the same portends a poor prognosis. Patients with PVTT generally are not suitable for TACE as the probability of hepatic decompensation with embolization is quite high in them. BCLC staging and treatment algorithm advises only the use of sorafenib for these patients who fall in BCLC Stage C. Lin et al. reported $79 \%$ response rates for PVTT in their prospective study on 43 patients who received RT for PVTT. ${ }^{[20]}$ They also reported survival benefit for those who responded. In another study ${ }^{[21]}$ of 41 patients treated with SBRT for PVTT and/or inferior vena cava thrombus, the authors reported $36.6 \%$ complete response rates and $39 \%$ partial response rates of PVTT. They too reported survival benefit to patients who responded. $\mathrm{Li}$ et al. recently reported results on 108 pairs of patients with PVTT who were paired based on propensity score matching. These pairs compared patients receiving TACE + SBRT versus TACE alone. The median survival time was 10.9 versus 4.1 months $(P<0.001)$ in all patients, 12.5 versus 4.4 months $(P=0.002)$ in patients with PVTT involving the right/left portal vein, and 8.9 versus 4.0 months $(P<0.001)$ in patients with PVTT involving the main portal vein trunk. At uni- and multivariate analysis, SBRT + TACE as treatment, maximum lesion diameter and PVTT in the main trunk were the independent prognostic factors for survival. It is reported that TACE and SBRT yield better outcomes when planned in succession than as salvage; ${ }^{[18]}$ however, which modality should be employed first is a matter of debate. A randomized study by Kang et al. ${ }^{[22]}$ where they randomized patients into SBRT followed by TACE (Group A), TACE followed SBRT (Group B), and SBRT alone (Group $\mathrm{C}$ ) reported overall response rate of $87.1 \%$. Between Groups A and B, the differences in the response rate, survival rate, $\alpha$-fetoprotein level restoration rate, and rate of improvement of abdominal distention and discomfort were not statistically significant $(P>0.05)$. However, these rates for Groups A and B were significantly higher compared to those of Group $\mathrm{C}(P<0.05)$. Importantly, deterioration of liver function in Group A was significantly lower compared to that in Group B $(P<0.05)$. The authors reported that compared to SBRT followed by TACE versus SBRT alone, TACE followed by SBRT may exert a negative effect on liver function. ${ }^{[22]}$ Yoon et al. reported the results of the START trial in 2018. They randomized $90 \mathrm{CP}-\mathrm{A}$ patients with liver-confined HCC and PVTT to either sorafenib or TACE followed by RT (TACE-RT). They reported results of TACE-RT versus sorafenib in 12-week progression-free survival (87\% vs. 34\%), 24-week radiographic response rate (33\% vs. $2 \%)$, median time to progression (31 vs. 12 weeks), and OS (55 vs. 43 months). In this disease with an overall grim prognosis, these results are quite encouraging toward a strong consideration for SBRT in multimodality treatment of HCC.

\section{Stereotactic Body Radiation Therapy as a Bridge to Transplant}

TACE, RFA, and SBRT are all utilized as a bridge to transplantation. A retrospective cohort analysis at Princess Margaret Hospital reported no survival difference between these three modalities in patients treated as a bridge to transplantation. ${ }^{[23]}$ While only $24 \%$ and $36 \%$ of TACE and SBRT patients met the Milan criteria, $88 \%$ of RFA patients met these criteria. A recent study presented at the 2017 GI Cancers Symposium reported outcomes of prospective comparison between SBRT and TACE for transplant patients. ${ }^{[24]}$ Time to retreatment was their primary endpoint, which is important in this clinical setting. They reported $40 \%$ rates of retreatment in TACE arm compared to none in SBRT arm. One of the secondary endpoints reported was toxicity that was found to be more in the TACE group.

All the clinical data shared above suggest that SBRT is an effective local therapy for parenchymal lesions as well as PVTT. The combination of TACE and SBRT becomes relatively more effective with higher survival rates. In the presence of PVTT, SBRT should precede 
TACE. This approach is rational and understandable because recanalization post-SBRT can improve outcomes post-TACE by providing better perfusion of normal liver when the hepatic artery is embolized. It is also evident that in the light of recent results of START trial, ${ }^{[22]}$ TACE and radiation therapy must be strongly considered for better outcomes as compared with sorafenib alone.

SBRT, which is newer modality, is different from other LDTs in being biological in its approach, because it targets the ultrastructural DNA damage and change of tumor microenvironment. It does not rely on physical damage to the tumor and therefore is effective in areas close to blood vessels, liver capsule, in lesions that are irregular or not well demarcated, in lesions with PVTT, and in relatively larger lesions $(>2 \mathrm{~cm})$. SBRT can also be effective in unresectable lesions, as a bridge therapy, along with TACE (before or after depending upon the presence of PVTT) for superior outcomes. For PVTT, it is a highly effective modality that improves survival, especially in patients with better hepatic reserves. It is now prudent to perform and report randomized trials with LDTs for better understanding of their relative merits.

Financial support and sponsorship

Nil.

\section{Conflicts of interest}

There are no conflicts of interest.

\section{Gagan Saini}

Department of Radiation Oncology, Max Institute of Cancer Care, Delhi NCR, India

Address for correspondence: Dr. Gagan Saini,

Department of Radiation Oncology, Max Institute of Cancer Care, Max Vaishali and Patparganj, Delhi NCR, India. E-mail: drgagansaini@gmail.com

Submitted: $17-\mathrm{Feb}-2020$

Revised: 05-Apr-2020

Accepted: 01-Jun-2020

Published: 29-Aug-2020

\section{References}

1. Tummala KS, Brandt M, Teijeiro A, Graña O, Schwabe RF, Perna C, et al. Hepatocellular carcinomas originate predominantly from hepatocytes and benign lesions from hepatic progenitor cells. Cell Rep 2017;19:584-600.

2. Acharya SK. Epidemiology of hepatocellular carcinoma in India. J Clin Exp Hepatol 2014;4:S27-33.

3. Kumar R, Kumar Saraswat M, Chander Sharma B, Sakhuja P, Sarin SK. Characteristics of hepatocellular carcinoma in India: A retrospective analysis of 191 cases. QJM 2008;101:479-85.

4. Guckenberger $\mathrm{M}$, Andratschke $\mathrm{N}$, Alheit $\mathrm{H}$, Holy $\mathrm{R}$, Moustakis C, Nestle U, et al. Definition of stereotactic body radiotherapy: Principles and practice for the treatment of stage I non-small cell lung cancer. Strahlenther Onkol 2014;190:26-33.

5. Wahl DR, Stenmark MH, Tao Y, Pollom EL, Caoili EM, Lawrence TS, et al. Outcomesww after stereotactic body radiotherapy or radiofrequency ablation for hepatocellular carcinoma. J Clin Oncol 2016;34:452-9.

6. Rajyaguru DJ, Borgert AJ, Smith AL, Thomes RM, Conway PD, Halfdanarson TR, et al. Radiofrequency ablation versus stereotactic body radiotherapy for localized hepatocellular carcinoma in nonsurgically managed patients: Analysis of the national cancer database. J Clin Oncol 2018;36:600-8.

7. Bibault JE, Dewas S, Vautravers-Dewas C, Hollebecque A, Jarraya $\mathrm{H}$, Lacornerie $\mathrm{T}$, et al. Stereotactic body radiation therapy for hepatocellular carcinoma: Prognostic factors of local control, overall survival, and toxicity. PLoS One 2013;8:e77472.

8. Andolino DL, Johnson CS, Maluccio M, Kwo P, Tector AJ, Zook J, et al. Stereotactic body radiotherapy for primary hepatocellular carcinoma. Int $\mathrm{J}$ Radiat Oncol Biol Phys 2011;81:e447-53.

9. Jung J, Yoon SM, Kim SY, Cho B, Park JH, Kim SS, et al. Radiation-induced liver disease after stereotactic body radiotherapy for small hepatocellular carcinoma: Clinical and dose-volumetric parameters. Radiat Oncol 2013;8:249.

10. Sanuki N, Takeda A, Mizuno T, Oku Y, Eriguchi T, Iwabuchi S, et al. Tumor response on $\mathrm{CT}$ following hypofractionated stereotactic ablative body radiotherapy for small hypervascular hepatocellular carcinoma with cirrhosis. AJR Am J Roentgenol 2013;201:W812-20.

11. Higgins H, Berger DL. RFA for liver tumors: Does it really work? Oncologist 2006;11:801-8.

12. Méndez Romero A, Wunderink W, Hussain SM, De Pooter JA, Heijmen BJ, Nowak PC, et al. Stereotactic body radiation therapy for primary and metastatic liver tumors: A single institution phase i-ii study. Acta Oncol 2006;45:831-7.

13. Kang JK, Kim MS, Cho CK, Yang KM, Yoo HJ, Kim JH, et al. Stereotactic body radiation therapy for inoperable hepatocellular carcinoma as a local salvage treatment after incomplete transarterial chemoembolization. Cancer 2012;118:5424-31.

14. Bujold A, Massey CA, Kim JJ, Brierley J, Cho C, Wong RK, et al. Sequential phase I and II trials of stereotactic body radiotherapy for locally advanced hepatocellular carcinoma. J Clin Oncol 2013;31:1631-9.

15. Culleton S, Jiang H, Haddad CR, Kim J, Brierley J, Brade A, et al. Outcomes following definitive stereotactic body radiotherapy for patients with Child-Pugh B or C hepatocellular carcinoma. Radiother Oncol 2014;111:412-7.

16. Scorsetti M, Comito T, Cozzi L, Clerici E, Tozzi A, Franzese $\mathrm{C}$, et al. The challenge of inoperable hepatocellular carcinoma (HCC): Results of a single-institutional experience on stereotactic body radiation therapy (SBRT). J Cancer Res Clin Oncol 2015;141:1301-9.

17. Huo YR, Eslick GD. Transcatheter arterial chemoembolization plus radiotherapy compared with chemoembolization alone for hepatocellular carcinoma: A systematic review and metaanalysis. JAMA Oncol 2015;1:756-65.

18. Buckstein M, Kim E, Fischman A, Blacksburg S, Facciuto M, Schwartz M, et al. Stereotactic body radiation therapy following transarterial chemoembolization for unresectable hepatocellular carcinoma. J Gastrointest Oncol 2018;9:734-40.

19. Hoekstra J, Janssen HL. Vascular liver disorders (II): Portal vein thrombosis. Neth J Med 2009;67:46-53.

20. Lin CS, Jen YM, Chiu SY, Hwang JM, Chao HL, Lin HY, et al. Treatment of portal vein tumor thrombosis of hepatoma patients with either stereotactic radiotherapy or three-dimensional conformal radiotherapy. Jpn J Clin Oncol 2006;36:212-7.

21. Xi M, Zhang L, Zhao L, Li QQ, Guo SP, Feng ZZ, et al. Effectiveness of stereotactic body radiotherapy for hepatocellular 
carcinoma with portal vein and/or inferior vena cava tumor thrombosis. PLoS One 2013;8:e63864.

22. Kang J, Nie Q, DU R, Zhang $\mathrm{L}$, Zhang J, Li Q, et al. Stereotactic body radiotherapy combined with transarterial chemoembolization for hepatocellular carcinoma with portal vein tumor thrombosis. Mol Clin Oncol 2014;2:43-50.

23. Sapisochin G, Barry A, Doherty M, Fischer S, Goldaracena N, Rosales R, et al. Stereotactic body radiotherapy vs. TACE or RFA as a bridge to transplant in patients with hepatocellular carcinoma. An intention-to-treat analysis. J Hepatol 2017;67:92-9.

24. Nugent FW, Qamar A, Stuart KE, Galuski K, Flacke S, Molgaard C, et al. A randomized phase II study of individualized stereotactic body radiation therapy (SBRT) versus transarterial chemoembolization (TACE) with DEBDOX beads as a bridge to transplant in hepatocellular carcinoma (HCC). J Clin Oncol 2017;35(suppl 4S; abstract 223):223.
This is an open access journal, and articles are distributed under the terms of the Creative Commons Attribution-NonCommercial-ShareAlike 4.0 License, which allows others to remix, tweak, and build upon the work non-commercially, as long as appropriate credit is given and the new creations are licensed under the identical terms.

For reprints contact: WKHLRPMedknow_reprints@wolterskluwer.com

\begin{tabular}{|l|l|}
\hline \multicolumn{2}{|c|}{ Access this article online } \\
\hline Quick Response Code: & Website: \\
& www.ijmpo.org \\
\cline { 2 - 3 } & DOI: \\
\hline
\end{tabular}

How to cite this article: Saini G. Stereotactic body radiation therapy in hepatocellular carcinoma. Indian J Med Paediatr Oncol 2020;41:488-91. 